\title{
Plasmonic coupling in Au, Ag and Al nanosphere homo-dimers for sensing and SERS
}

\author{
Jyoti Katyal \\ Amity Institute of Applied Science, Amity University, Noida, UP, India \\ *Corresponding author, E-mail: jyoti_106@yahoo.co.in
}

\begin{abstract}
The localized surface plasmon resonance of homodimer nanostructures is studied using FDTD simulations. The calculated LSPR wavelength of Au, $\mathrm{Ag}$ and $\mathrm{Al}$ nanosphere forming a homo-dimer configuration is compared and the results reveal a larger LSPR shift in $\mathrm{Ag}$ and $\mathrm{Al}$ homo-dimer than in Au homo-dimer. Taking the sensitivity of LSPR shape to the size and interparticle spacing of nanoparticle along with a surrounding refractive index, parameters like refractive index sensitivity have been determined. The spherical homo-dimer over the whole range of particle size, studied here shows the index sensitivity order as $\mathrm{Ag}>\mathrm{Al}>\mathrm{Au}$. Hence, the use of plasmonic material towards the refractive index sensing applications is useful in this order. The average refractive index sensitivities of $\mathrm{Ag}, \mathrm{Al}$ and $\mathrm{Au}$ are $287.09 \mathrm{~nm} / \mathrm{RIU}, 210.21 \mathrm{~nm} / \mathrm{RIU}$ and $192.47 \mathrm{~nm} / \mathrm{RIU}$ in DUV-Visible-NIR region. Apart from LSPR shift, the highly confined near-field intensity enhancement of homo-dimer nanostructures for SERS has also been studied. The interacting homo-dimer nanoparticles reveals intensity enhancements in the junction. Comparing the field enhancement for $\mathrm{Au}, \mathrm{Ag}$ and $\mathrm{Al}$ homo-dimer nanostructure $\sim 10^{8}-10^{9}$ have been theoretically predicted in DUV-UV-visible region which can be used to strongly enhance the Raman scattering of molecules.
\end{abstract}

Keywords: Localised surface plasmon resonance, Homo-dimer, near-field enhancement, FDTD.

\section{Introduction}

When the metallic nanoparticle is subject to light excitation, the electric field of the light induces waves of collective electron oscillations which is confined to the surface of the nanoparticle. This phenomenon is known as a localized surface plasmon resonance (LSPR). In metallic particle, the frequency, strength and quality of the LSPR depends sensitively on the size, geometry, the metal composition and the refractive index of the local environment $[1,2]$. Another factor that defines the sensitivity of LSPR is the strongly interacting nanoparticle pairs. It is well accepted from the work of Nordlander and Halas and the El-Sayed group that plasmon coupling in homo dimer nanostructure can be viewed as analogous to molecular hybridization [3-6]. The plasmon modes ( $\Psi 1$ and $\Psi 2$ ) of two interacting metal nanoparticles hybridize either in-phase $(\Psi 1+\Psi 2)$ or out-of-phase $(\Psi 1-\Psi 2)$. When the incident light field is polarized along the interparticle axis (longitudinal polarization), the in-phase combination reflects a bonding mode (with electric field enhanced at the junction and a redshifted LSPR frequency) and the out-of phase mode represents an antibonding configuration (with the electric field localized on the non-junction ends of the particles and a blue-shifted LSPR frequency), respectively denoted as $\sigma$ and $\sigma^{*}$. However, when the polarization is perpendicular to the interparticle axis (transverse polarization), the scenario is exactly reversed, that is, the in-phase combination is an antibonding mode $\left(\pi^{*}\right)$ and the out-of phase one is a bonding mode $(\pi)$. In the case of plasmonic "homodimers", the antiphase mode is spectrally dark due to the cancellation of the equal but oppositely oriented dipoles on the two particles. Hence, a homodimer structure under longitudinal polarization supports only a bonding Plasmon mode $(\sigma)$ with its electric field strongly localized at the junction of the dimer. Likewise, only a $\pi^{*}$-mode is observed in a homodimer under transverse polarization. Recently, a split nanoring dimer resonator which supports multiple plasmonic Fano-like resonances that arises from the coupling and interference of the dimer plasmon modes offers high values of figure of merit and contrast ratio due to which they are suitable for high-performance biological sensors [7]. The theoretically investigated plasmonic Fano resonance in a triangular nanoprism dimer shows FOM as high as 16.1 in visible and NIR region due to large electric field enhancement at the corners of the nanoprisms [8]. Acimovic et. al experimentally verified the exponentially increasing sensitivity enhancement in dimers of gold nanodiscs [9]. They employ nanoparticle dimers with small gaps for highly enhanced detection of proteins, with sensitivity tending towards single molecule detection. Apart from gold nanoparticles, the LSPR based sensor has been extended to UV wavelength [10,11, 12].

In the present work, we employed the FDTD simulation to dimer nanostructure for metallic nanoparticle over DUV- visible-NIR region. Figure 1 
shows a schematic diagram of metallic homo-dimer nanostructure. The particle size is D (diameter) and the interparticle spacing between two nanospheres forming a dimer is $\mathrm{d}$. The nanosphere is assumed to be surrounded by vacuum. The particle is illuminated with an incident light along xy-plane and electric field polarized along $\mathrm{x}$-direction, as indicated in figure 1 by symbol $\mathrm{k}$ and E, respectively. We present a theoretical comparison of the LSPR shift and the associated near field confinement caused by the coupling of different plasmonic modes for $\mathrm{Au}, \mathrm{Ag}$ and $\mathrm{Al}$ homo-dimer nanostructure given by parameter $\eta$. We found that due to strong field confinement, a plasmonic refractive index sensor with high sensitivity can be realized over DUV-Visible-NIR region by choosing the proper material. The near field calculation shows that a remarkably uniform field enhancement occurs at the junction of homo-dimer nanostructure, which can strongly enhance the Raman scattering of molecules lying in these junctions.

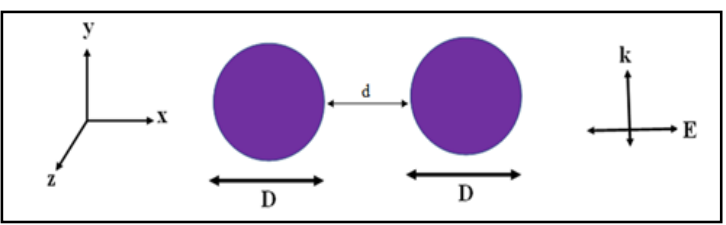

Figure 1: Schematic of a homo-dimer metallic nanosphere. The direction of propagation of EM field incident on the sphere is y-direction and field is polarized along the dimer axis ( $\mathrm{x}$-axis).

\section{Theoretical Simulations}

In this work, we employed FDTD software provided by Lumerical FDTD solutions to calculate optical spectra of a homo-dimer nanosphere as shown in figure 1 [12]. We adopt a cubic Yee cell with a side equal to $1 \mathrm{~nm}$ and a time step $\Delta \mathrm{t}=1.31 \times 10^{-18} \mathrm{~s}$, bounded by Courant condition. For FDTD calculations involving the nanosphere the grid size is selected in such a way that the E-field intensity around nanospheres becomes independent of the grid size [13]. This process was carried out by initially selecting a grid size of $1 / 10^{\text {th }}$ of nanosphere size and subsequently reducing the grid spacing until a further reduction in grid spacing had no effect on the calculated values around the nanosphere. The FDTD code allows different choice of grid size near the edge of nanostructure, we used $0.7 \mathrm{~nm}$ grid spacing around the nanosphere. The material data used is fitted to have a larger index. The material data were obtained from $\mathrm{J} \& \mathrm{C}$ and Palik for $\mathrm{Au}$ and $\mathrm{Ag}$, respectively, whereas for $\mathrm{Al}$ in wavelength region $>200 \mathrm{~nm}$ was taken from Palik and in a wavelength region $\leq 200$ nm from CRC model [11,12].

\section{Results \& Discussion}

The simulated optical spectra of $\mathrm{Au}$ homo-dimer nanostructure, interacting along dimer axis is shown in figure 2. The particle size (D) is $20 \mathrm{~nm}$ and interparticle spacing is varied as $1 \mathrm{~nm}$ to $10 \mathrm{~nm}$. For an interparticle spacing $10 \mathrm{~nm}$, the peak wavelength is observed at $512 \mathrm{~nm}$, which is close to isolated $\mathrm{Au}$ nanosphere. The plasmon peak shifts to $521 \mathrm{~nm}$ and $536 \mathrm{~nm}$ for interparticle spacing $3 \mathrm{~nm}$ and $1 \mathrm{~nm}$, respectively. The smaller the gap between the particles, the larger is the red-shift. In the same manner, the dipole plasmon wavelength has been evaluated for $\mathrm{Ag}$ and $\mathrm{Al}$ homo dimer nanostructure. Figure 3(a) compares the peak wavelength for $\mathrm{Au}, \mathrm{Ag}$ and $\mathrm{Al}$ homo-dimer nanostructure having particle size as $20 \mathrm{~nm}$ and $40 \mathrm{~nm}$. The general trend i.e., the red shift in plasmon resonance wavelength with decreasing interparticle spacing is observed for nanodimer configuration which increases almost exponential with the reduction in interparticle spacing. When particles come closer to each other because of the near-field coupling of the plasmons, there is a change in the frequency of the surface plasmon oscillation of the coupled nanoparticle system with respect to the isolated particle. For parallel polarization, the magnitude of the force increases strongly with decreasing spacing between particles. This is a direct consequence of the increasing fieldenhancement between the particles. At the same time, the spectral position of the force maximum red-shifts due to plasmon hybridization [3].

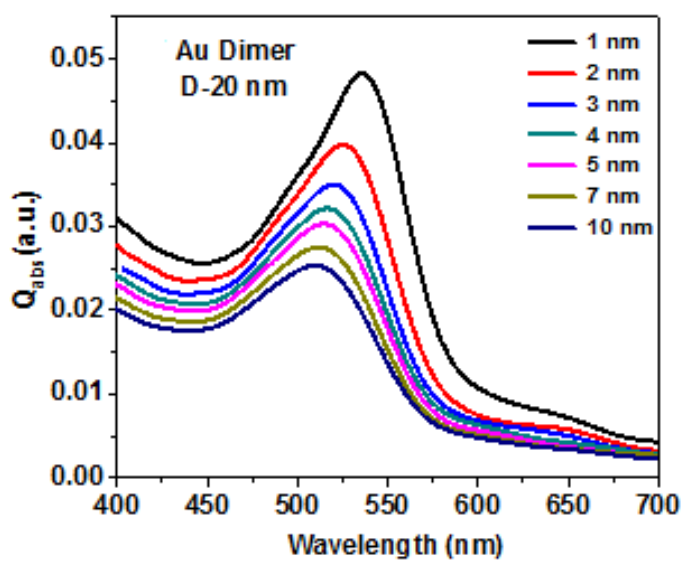

Figure 2: Absorption spectra of Au homo dimer nanostructure. The particle size is $20 \mathrm{~nm}$ and spacing is varied from $1 \mathrm{~nm}$ to $10 \mathrm{~nm}$. 

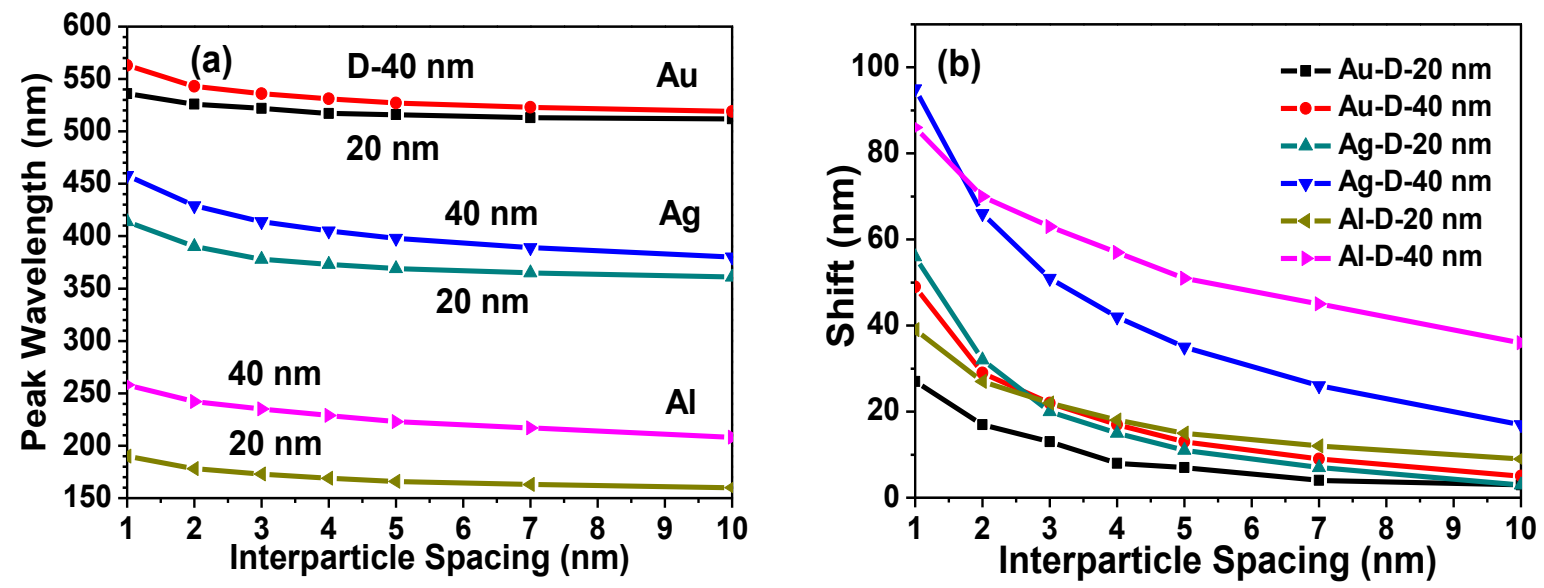

Figure 3: (a) Variation of dipole resonance wavelength of $\mathrm{Au}, \mathrm{Ag}$ and $\mathrm{Al}$ homo-dimer with interparticle spacing. (b) The LSPR wavelength shift of homo-dimer nanostructure wrt isolated nanosphere with interparticle spacing. The particle size is $20 \mathrm{~nm}$ and $40 \mathrm{~nm}$.

Figure 3 (b) compares the calculated shift in peak wavelength from an isolated nanosphere to dimer nanostructure depending on interparticle spacing. It can be observed that $\mathrm{Al}$ shows stronger coupling as compared to $\mathrm{Ag}$ and $\mathrm{Au}$ which results in larger shift. With $10 \mathrm{~nm}$ interparticle spacing there is still a stronger coupling taking place between two identical Al nanospheres $(\mathrm{D}-40 \mathrm{~nm})$ forming a dimer giving 36 $\mathrm{nm}$ as a shift in plasmon wavelength whereas Au and Ag show much lower value. This is because of the large field experienced by dipoles in $\mathrm{Al}$ as compared to $\mathrm{Au}$ and $\mathrm{Ag}$ nanodimer configuration. With stronger coupling in $\mathrm{UV}$-visible region $\mathrm{Al}$ can replace $\mathrm{Au}$ and Ag nanoparticle in various applications like solar cell, UV based SERS etc $[14,15]$.

A plasmon rule which states that regardless of the type of the metal, the shape of the particle, or the surrounding medium, the near-field coupling depth is always $\sim 0.2$ times the diameter providing a universal scaling behavior of plasmon coupling is studied for $\mathrm{Al}$ dimer to validate our results [3]. Figure 4 shows the calculated resonance wavelength of the dimer fitted to the plasmon rule equation. From the plot, an exponential decay constant of $0.19 \pm 0.02$ is estimated for $\mathrm{Al}$ dimer. The value of decay constant is close to that observed for the universal scaling trend in the plasmon coupling-inducing shift of the nanosphere pair as a function of interparticle spacing scaled by particle size.



Figure 4: Fractional plasmon shift versus the ratio of spacing to the particle size for Al homo-dimer. The particle size is $20 \mathrm{~nm}$ and $40 \mathrm{~nm}$.

It is known from the plasmonics literature that greatly enhanced fields are induced by light incident on dimers [2,16,17]. Figure 5(a) shows the near-field pattern for a homo-dimer nanostructure. The incident field is along the axis of two nanospheres forming a homo-dimer configuration. The particle size is $20 \mathrm{~nm}$. When an optical radiation is incident on homo-dimer nanostructure then an oscillating polarization is induced in nanoparticles and if they are polarized in the same manner, i.e. opposite surface charges at both spheres, located at a short distance to each other, will produce a strong field. Maximum field is located in a small region where the two nanosphere interact with each other and a small increment in field enhancement is observed on the other faces of nanosphere. For particle size $20 \mathrm{~nm}$, field enhancement factor $(\eta)$ is 11 at resonance wavelength $522 \mathrm{~nm}$ corresponding to 

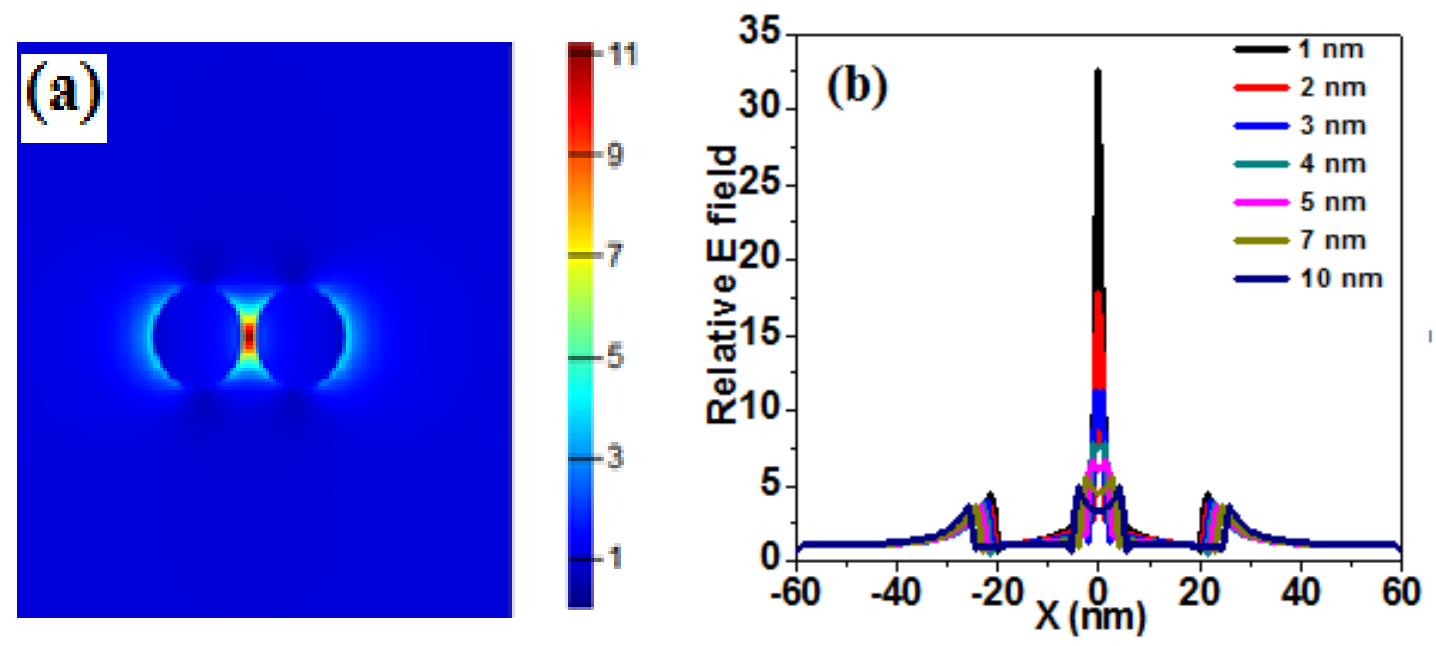

Figure 5: (a) The near-field pattern at a plasmon resonance wavelength in xz-plane for two identical Au nanospheres forming a homo-dimer nanostructure with particle size as $20 \mathrm{~nm}$. The interparticle spacing is $3 \mathrm{~nm}$. The image width is from $-60 \mathrm{~nm}$ to $60 \mathrm{~nm}$. The plasmon resonance wavelength is $522 \mathrm{~nm}$.(b) Interparticle spacing dependent $\eta$ along $\mathrm{x}$ - axis for Au homo-dimer nanostructure.

Au homo-dimer nanostructure, figure 5 (a).

Figure 5(b) shows the variation in relative electric field, along the $\mathrm{x}$-axis with interparticle spacing. The enhancement factor shows maxima between the two nanosphere, whereas at the outer surface it is not much higher as in isolated nanosphere where maximum enhancement is observed at the outer surface of nanosphere. With $\mathrm{Au}$ as plasmonic material, the enhancement is occurring for wavelengths $>510 \mathrm{~nm}$. This open window of plasmon resonance wavelength does not consider the lower wavelength region where biological molecules shows absorption, therefore, to explore the near-field enhancement toward DUV and UV region we considered the Ag and Al homo-dimer nanostructure. The near field pattern of $\mathrm{Ag}$ and $\mathrm{Al}$ homo-dimer nanostructure for particle size $20 \mathrm{~nm}$ and $40 \mathrm{~nm}$ are evaluated over resonance wavelength. Figure 6 compares the size dependent and interparticle spacing dependent enhancment factor for three different materials as $\mathrm{Au}, \mathrm{Ag}$ and $\mathrm{Al}$. The $\eta \sim 31$ with $\mathrm{Ag}$ homo-dimer (D- $20 \mathrm{~nm}$ ) at resonance wavelength $378 \mathrm{~nm}$ which increases to $\sim 90$ with particle size as $40 \mathrm{~nm}$. Apart from Ag homo-dimer, Al homo-dimer shows higher enhancement factor with small particle size. The near-field pattern for Al nanospheres forming a homo-dimer configuration shows that $\eta$ increases to $\sim 43$ for particle size $20 \mathrm{~nm}$ at $172 \mathrm{~nm}$. Such a huge enhancement in UV region makes $\mathrm{Al}$ as a material for UV-SERS [15]. It is known that $\mathrm{Al}$ shows the number of peaks with increasing particle size which reduces the enhancement factor due to which
$\mathrm{Al}$ shows opposite behavior as compared to $\mathrm{Au}$ and Ag. The $\eta$ increases from 11 to 47.96 on increasing the particle size of Au homo-dimer to $50 \mathrm{~nm}$, whereas Ag shows much higher $\eta \sim 110$. For larger particle size Al nanoparticle shown the contribution of multipole peaks, therefore, the $\eta$ decreases to 36 as particle size increases to $50 \mathrm{~nm}$. The enhancement can further be increased as particle approaches each other shown in figure 6(b). The $\eta$ shows maximum field enhancement $\sim 200$ in UV-visible region for $\mathrm{Ag}$ homo-dimer, whereas Al homo-homo-dimer shows 120 in DUV-UV region with interparticle spacing as 1 $\mathrm{nm}$. The value of the electromagnetic enhancement is proportional to the fourth power of the localized field intensity at the surface of the SERS active surface [1618]. It is, therefore, important to theoretically estimate the EM field enhancement for homo dimer nanostructure forming the SERS substrates so that substrates with extremely high enhancement factors can be designed and fabricated. As compared to isolated nanoparticle, the interacting nanoparticle shows large enhancement factor over a wider range of plasmon wavelength which shows their potential to be used as a SERS substrate. An Al nanosphere shows enhancement up to $120\left(\sim 10^{8}\right)$ for dimer configuration whereas Ag nanosphere shows enhancement upto 200 $\left(\sim 10^{9}\right)$ in Deep UV and UV-visible region, respectively. This potential can be used to strongly enhance the Raman scattering of molecules lying in these junctions. 

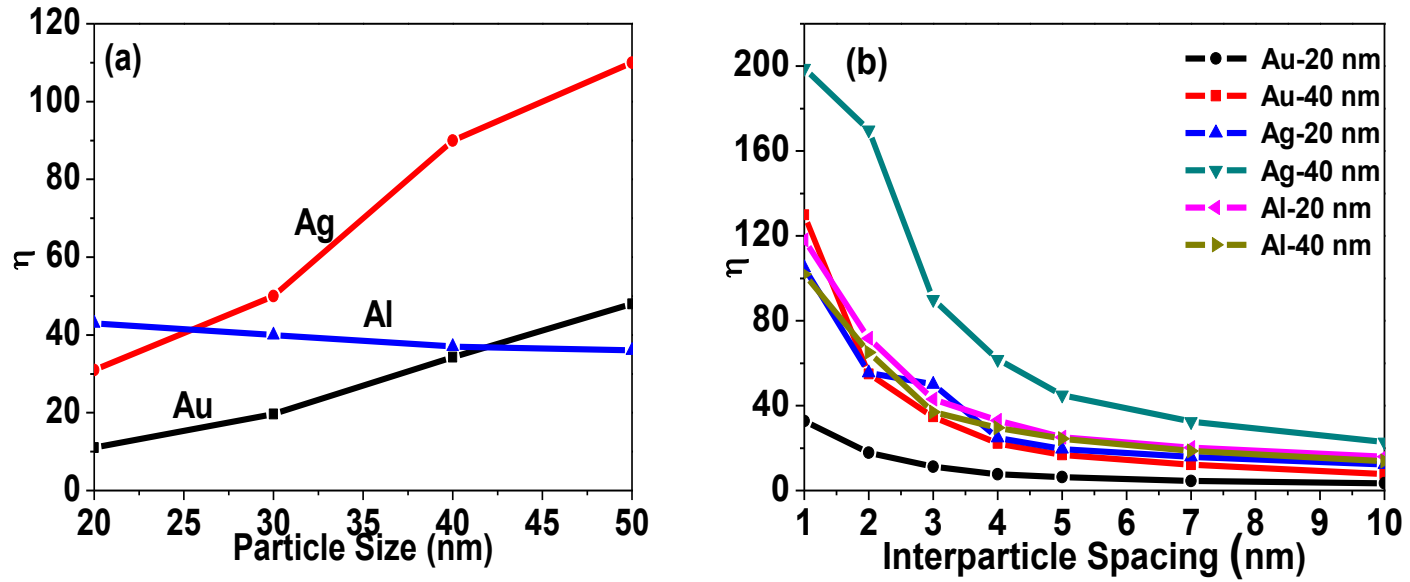

Figure 6: (a) Size dependent and (b) Interparticle spacing dependent $\eta$ for homo-dimer nanostructure.
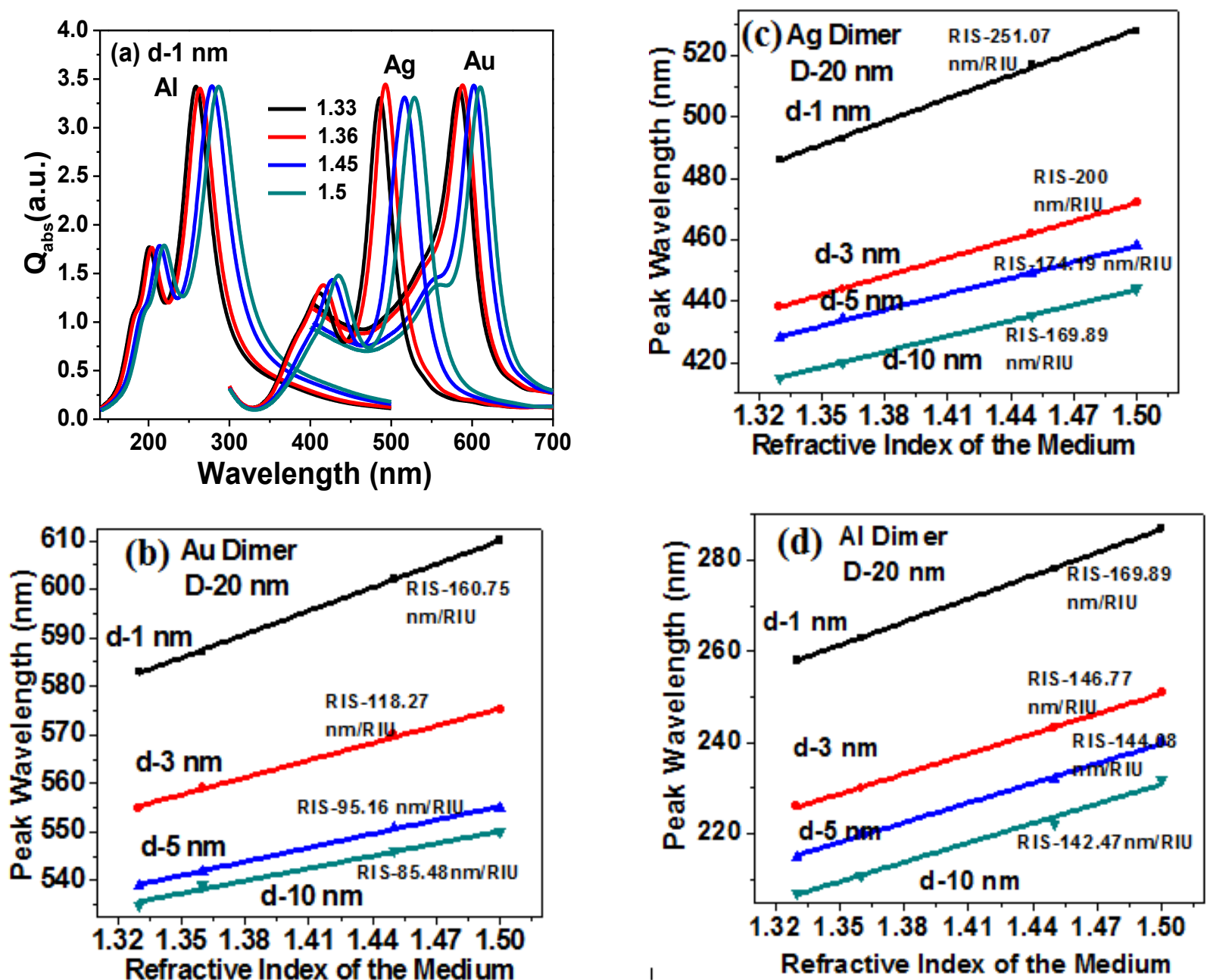

Figure 7: (a) Calculated absorption spectra of $\mathrm{Au}, \mathrm{Ag}$ and $\mathrm{Al}$ homo-dimer. The refractive index of the medium is $1.33,1.36,1.45$ and 1.5. The particle size is $20 \mathrm{~nm}$ and the interparticle spacing is $1 \mathrm{~nm}$. Interparticle spacing dependent shift in peak wavelength with refractive index of the medium for (b) Au, (c) Ag and (d) Al homo-dimer nanostructure. 
The sensitivity of the plasmon resonance wavelength on the surrounding medium dielectric constant is very useful in LSPR based sensing of chemical and biomolecular analytes [19,20] and it is defined in terms of refractive index sensitivity (RIS) as, RIS = $\frac{\mathrm{d} \lambda_{\mathrm{p}}}{\mathrm{dn}}$, where, $\mathrm{d} \lambda_{\mathrm{p}}$ is the shift in LSPR peak wavelength [21].

Figure 7(a) shows the calculated absorption spectra of $\mathrm{Au}, \mathrm{Ag}$ and $\mathrm{Al}$ nanosphere homo-dimer. The particle size is $20 \mathrm{~nm}$ with interparticle spacing as $1 \mathrm{~nm}$. The refractive index of the medium is fixed as $1.33,1.36$, 1.45 and 1.5. A red shift in plasmon wavelength is observed as the refractive index of the medium is increased. The spectra is evaluated for $\mathrm{Au}, \mathrm{Ag}$ and $\mathrm{Al}$ nanosphere homo-dimer by varying interparticle spacing. Figure 7(b), (c) and (d) shows linearly fitted graph between peak wavelength and refractive index for $\mathrm{Au}, \mathrm{Ag}$ and $\mathrm{Al}$, respectively. The fitting parameters $\mathrm{x}$ and $\mathrm{y}$ of linear equation $\lambda_{\mathrm{p}}=\mathrm{y} \cdot \mathrm{n}+\mathrm{x}$ are given in Table 1. Figure 8 shows calculated shift of dipole resonance peak wavelength with refractive index of the medium. The regression analysis of $\lambda_{p}$ yields the refractive index sensitivity (RIS) in unit of $\mathrm{nm} / \mathrm{RIU}$. It can be seen that RIS factor increases rapidly as the interparticle separation in the homodimer nanostructure is decreased irrespective of metal. The Au homo-dimer shows RIS factor of 160.75 $\mathrm{nm} / \mathrm{RIU}$ which decreases to $118.27 \mathrm{~nm} / \mathrm{RIU}$ on increasing interparticle spacing from $1 \mathrm{~nm}$ to $3 \mathrm{~nm}$. On further increasing the interparticle separation to 10 $\mathrm{nm}$, the homo-dimer start acting as individual nanosphere, therefore, their RIS factor further decreases to $85.48 \mathrm{~nm} / \mathrm{RIU}$ i.e., start approaching the value of individual $\mathrm{Au}$ nanosphere of the same size. The Au homo-dimer with particle size $40 \mathrm{~nm}$ also shows a similar behavior. The index sensitivity increases from $77 \mathrm{~nm} / \mathrm{RIU}$ to $172 \mathrm{~nm} / \mathrm{RIU}$ as the interparticle separation decreases from $90 \mathrm{~nm}$ to $4 \mathrm{~nm}$ [22]. As compared to $\mathrm{Au}, \mathrm{Ag}$ and $\mathrm{Al}$ homo-dimer show larger value of RIS factor which is due to the difference in dielectric constant of three metals. Ag shows maxima of $251.07 \mathrm{~nm} / \mathrm{RIU}$ which decreases to $169.89 \mathrm{~nm} / \mathrm{RIU}$ with increasing interparticle separation, whereas Al shows maxima of 169.89 $\mathrm{nm} / \mathrm{RIU}$ which decreases to $142.47 \mathrm{~nm} / \mathrm{RIU}$ as interparticle separation increases to $10 \mathrm{~nm}$. The rate of decrease in RIS factor is minimum for Al homo-dimer as compared to $\mathrm{Au}$ and $\mathrm{Ag}$ homo-dimer, figure 8. The medium sensitivity is found to increase nearexponentially with a decrease in the interparticle spacing and the trend shown in figure 8 closely matches the universal scaling decay.

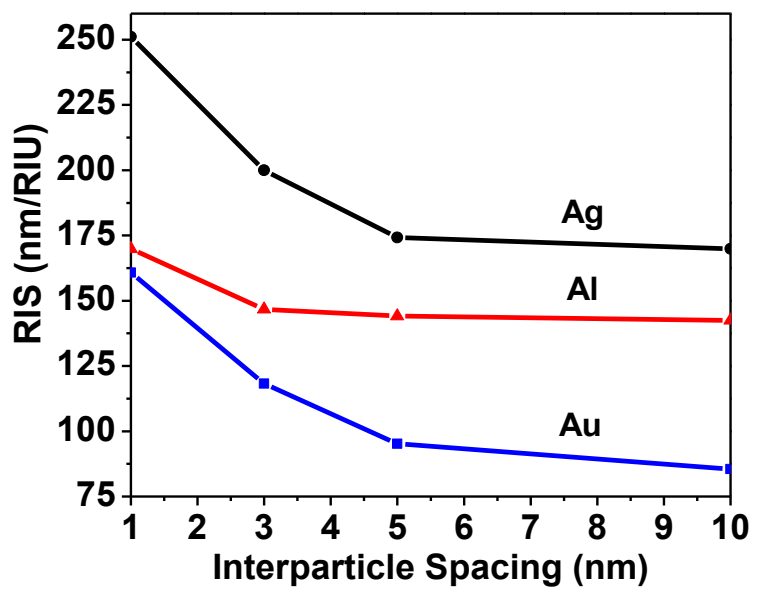

Figure 8: The interparticle spacing dependent refractive index sensitivity of $\mathrm{Au}, \mathrm{Ag}$ and $\mathrm{Al}$ homodimer nanostructure. The particle size is $20 \mathrm{~nm}$.

The refractive index sensitivity of isolated nanosphere of $\mathrm{Au}, \mathrm{Ag}$ and $\mathrm{Cu}$ calculated over different range of radius $(20 \mathrm{~nm}$ to $80 \mathrm{~nm}$ ) shows the sensitivity in the order as $\mathrm{Ag}>\mathrm{Au}>\mathrm{Cu}$ as $237.18>191.01>153.14 \mathrm{~nm} / \mathrm{RIU}$ [19]. Recently, the UV nanoplasmonics aluminum (Al) nanoconcave has shown the RIS factor of $\sim 191 \mathrm{~nm} /$ RIU with interpores distance $\left(D_{c}\right)$ of $246.3 \mathrm{~nm}$ and $291 \mathrm{~nm} / \mathrm{RIU}$ with $\mathrm{D}_{\mathrm{c}}$ of $456.7 \mathrm{~nm}$ [15]. Experimentally, fabricated $\mathrm{Au}$ nanodots and nanodisks having LSPR in visible region shows the RIS factor of $\sim 196 \mathrm{~nm} / \mathrm{RIU}$ and $\sim 226$ $\mathrm{nm} /$ RIU. The particle size is $180 \mathrm{~nm}$ and $\sim 150 \mathrm{~nm} \mathrm{[23,}$ $24,25]$. Our calculated result proves that homo-dimer nanostructure shows the higher RIS factor as compared to isolated nanoparticle or non-interacting array in DUV-visible- NIR region. 

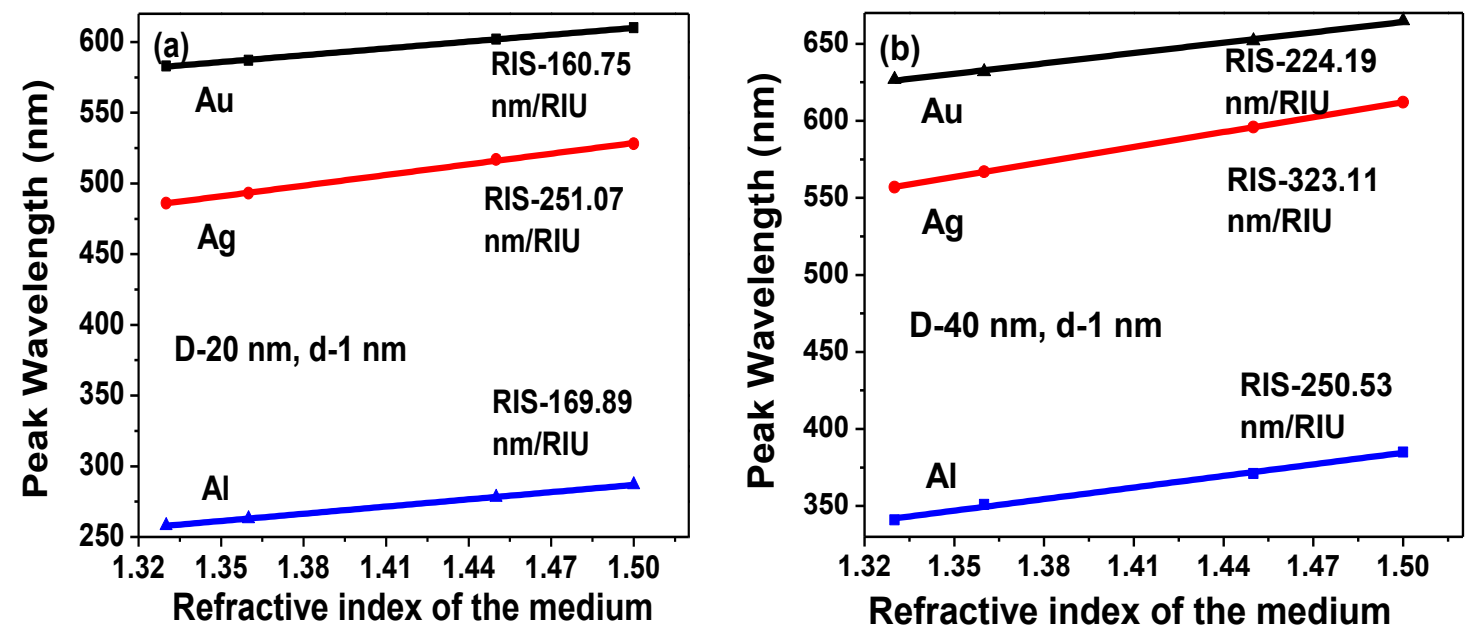

Figure 9: Calculated peak wavelength shift with refractive index of the medium for $\mathrm{Au}, \mathrm{Ag}$ and $\mathrm{Al}$ homo-dimer. The particle size is (a) $20 \mathrm{~nm}$ and (b) $40 \mathrm{~nm}$. The interparticle spacing is $1 \mathrm{~nm}$.

\section{Conclusion}

The LSPR properties and near field of Au, Ag and $\mathrm{Al}$ nanosphere homo-dimer in the DUV-NIR region is studied using FDTD simulations. The plasmon coupling leading to red shift of the plasmon wavelength depends on particle size, interparticle spacing and material. Comparison of the strength of coupling for $\mathrm{Au}, \mathrm{Ag}$ and $\mathrm{Al}$ particles with similar geometry shows the coupling order as $\mathrm{Al}>\mathrm{Ag}>\mathrm{Au}$. In addition, the highly confined near field enhancement is observed in homo-dimer nanostructure. Intensity enhancements in the junction of up to $\sim 10^{8}-10^{9}$ have been theoretically predicted in DUV-UV-visible region depending on the material. This property has attracted the homo-dimer nanostructure for many potential applications like SERS. Apart from this, the refractive index sensitivity is also found to be higher in homo-dimer nanostructure. Al nanostructure in DUV region, whereas $\mathrm{Au}$ and $\mathrm{Ag}$ shows higher sensitivity in larger wavelength region. The sensitivity not only depend on particle size, but also on interparticle spacing. $\mathrm{Ag}$ and $\mathrm{Al}$ homo-dimers show RIS factor as $323.11 \mathrm{~nm} / \mathrm{RIU}$ and $250.53 \mathrm{~nm} / \mathrm{RIU}$, respectively, which is higher as compared to 224.19 $\mathrm{nm} / \mathrm{RIU}$ for Au homo-dimer with interparticle spacing of $1 \mathrm{~nm}$. Comparison of different structures and materials for refractive index sensing reveals that dimer configuration is a superior structure than an isolated nanosphere or array of non-interacting particles and $\mathrm{Ag}$ or $\mathrm{Al}$ is superior material than $\mathrm{Au}$.

Table 1: Interparticle spacing dependent fitting parameters $\mathrm{x}$ and $\mathrm{y}$ of $\mathrm{Au}, \mathrm{Ag}$ and $\mathrm{Al}$ homo-dimer nanostructure.

\begin{tabular}{|c|c|c|c|c|c|c|c|c|c|c|c|c|}
\hline \multirow[b]{3}{*}{ d (nm) } & \multicolumn{4}{|c|}{ GOLD (Au) } & \multicolumn{4}{|c|}{ SILVER (Ag) } & \multicolumn{4}{|c|}{ ALUMINIUM (AI) } \\
\hline & \multicolumn{2}{|c|}{$\mathbf{x}$} & \multicolumn{2}{|c|}{$\mathbf{y}$} & \multicolumn{2}{|c|}{$\mathbf{x}$} & \multicolumn{2}{|c|}{$\begin{array}{l}\mathbf{y} \\
\end{array}$} & \multicolumn{2}{|c|}{$\mathbf{x}$} & \multicolumn{2}{|c|}{$\mathbf{y}$} \\
\hline & Value & S.E. & Value & S.E. & Value & S.E. & Value & S.E. & Value & S.E. & Value & S.E. \\
\hline $\mathbf{1}$ & 368.83 & 4.32 & 160.75 & 3.06 & 151.98 & 8.89 & 251.07 & 6.30 & 31.95 & 2.73 & 169.89 & 1.93 \\
\hline 3 & 397.97 & 5.35 & 118.27 & 3.79 & 172 & 0 & 200 & 0 & 30.54 & 4.05 & 146.77 & 2.87 \\
\hline 5 & 412.57 & 4.10 & 95.16 & 2.90 & 196.63 & 4.40 & 174.19 & 3.12 & 23.58 & 5.66 & 144.08 & 4.01 \\
\hline 10 & 421.96 & 7.60 & 85.48 & 5.38 & 188.95 & 2.73 & 169.89 & 1.93 & 17.11 & 15.43 & 142.47 & 10.93 \\
\hline
\end{tabular}




\section{References}

[1] K.L. Kelly, E. Coronado, L.L. Zhao, G.C. Schatz, The optical properties of metal nanoparticles: the influence of size, shape and dielectric environment, J. Phys. Chem. B,107 ( 2003 ) 668-677.

[2] C.F. Bohren, D.R. Huffman, Absorption and scattering of light by small particles, Wiley interscience publication, 1998.

[3] P.K. Jain, W. Huang, M.A. El-Sayed, On the universal scaling behavior of the distance decay of plasmon coupling in metal nanoparticle pairs: a plasmon ruler equation, Nano Lett.7 (2007) 20802088; P.K. Jain, M.A. El-Sayed, Plasmonic coupling in noble metal nanostructures, Chem. Phys. Lett. 487 (2010) 153-164.

[4] P. Nordlander, C. Oubre, E. Prodan, K. Li, M.I. Stockman, Plasmon hybridization in nanoparticle dimers, Nano Lett. 4 (2004) 899-903.

[5] E. Prodan, C. Radloff, N.J. Halas, P. Nordlander, A hybridization model for the plasmon response of complex nanostructures, Science 302(2003) 419422.

[6] Y. Gao, R. Zhang, J.C. Cheng, J.W. Liawc, C. Ma, Optical properties of plasmonic dimer, trimer, tetramer and pentamer assemblies of gold nanoboxes, Journal of Quantitative Spectrosc. \& Radiative Transfer 125 ( 2013) 23.

[7] Q.H. Wei, K.H. Su, S. Durant, X. Zhang, Plasmon resonance of finite one-dimensional Au nanoparticle chains, Nano Lett. 4 (2004) 1067-1071.

[8] H.P. Paudel, K. Bayat, M.F. Baroughi, S. May, D.W. Galipeau, Geometry dependence of field enhancement in 2D metallic photonic crystals, Optics Express 17(2009) 22179-22189.

[9] C.P. Burrows, W.L. Barnes, Large spectral extinction due to overlap of dipolar and quadrupolar plasmonic modes of metallic nanoparticles in arrays, Optics Express 18 (2010) 3187-3198.

[10] K. Li, M.I. Stockman, D.J. Bergman, Self-similar chain of metal nanospheres as an efficient nanolens, Phys. Rev. Lett. 91 (2003) 227402.

[11] G. Pellegrini, G. Mattei, V. Bello, P. Mazzoldi, Interacting metal nanoparticles: optical properties from nanoparticle dimers to core-satellite systems, Mater. Sci. Eng. C 27 (2007) 1347-1350.

[12] Lumerical Solutions; http://docs.lumerical.com.
[13]A. Taflove, S.C. Hagness, Computational Electrodynamics: The Finite-Difference TimeDomain Method, Norwood, MA: Artec House Publishers, 1995.

[14]N. Mattiucci, G. D’Aguanno, H.O. Everitt, J.V. Foreman, J.M. Callahan, M.C. Buncick, M.J. Bloemer, Ultraviolet surface-enhanced Raman scattering at the plasmonic band edge of a metallic grating, Optics Express 20 (2012) 1868-1877.

[15]M. Norek, M. Włodarski, P. Matysik, UV plasmonic-based sensing properties of aluminum nanoconcave arrays, Current Applied Physics 14 (2014) 1514-1520.

[16]U.K. Sur, J. Chowdhury, Surface-enhanced Raman scattering: overview of a versatile technique used in electrochemistry and nanoscience, Current Science, 105 (2013) 923.

[17]K.C. Bantz, A.F. Meyer, N.J. Wittenbergb, H. Imb, O. Kurtulusa, S.H. Leec, N.C. Lindquistb, S.H. Ohb, C.L. Haynesa, Recent progress in SERS biosensing, Phys. Chem. Chem. Phys., 13 (2011) 11551.

[18] M. Kall, H. Xu, P. Johansson, Field enhancement and molecular response in surface enhanced Raman scattering and fluorescence spectroscopy. Journal of Raman spectroscopy. 36 (2005) 510-514.

[19] M.H. Chowdhury, K. Ray, M.L. Johnson, S.K. Gray, J. Pond, J.R. Lakowicz, On the feasibility of using the intrinsic fluorescence of nucleotides for DNA sequencing, J. Phys. Chem. C 114 (2010) 7448-61.

[20] M.H. Chowdhury, K. Ray, S.K. Gray, J. Pond, J.R. Lakowicz, The use of Aluminum nanostructures as platforms for metal enhanced fluorescence of the intrinsic emission of biomolecules in the ultra-violet, Proc. of SPIE 7577 (2010) 757700-1.

[21] J.S. Sekhon, S.S. Verma, Refractive index sensitivity analysis of $\mathrm{Ag}, \mathrm{Au}$, and $\mathrm{Cu}$ nanoparticles, Plasmonics 6 (2011) 311-317.

[22]P.K. Jain, M.A. El-Sayed Noble metal nanoparticle pairs: effect of medium for enhanced nanosensing, Nano Lett. 8 (2008) 4347-4352.

[23] Y. Lin, Y. Zou, R.G. Lindquist, A reflection-based localized surface plasmon resonance fiber-optic probe for biochemical sensing Biomed., Opt. Express 2 (2011) 478-484.

[24] Y. Lin, Y. Zou, Y. Mo, J. Guo, R.G. Lindquist, Ebeam patterned gold nanodot arrays on optical fiber tips for localized surface plasmon resonance biochemical sensing, Sensors 10 (2010) 9397-9406. 